ARBOR Ciencia, Pensamiento y Cultura Vol. 187 - Extra 3 - diciembre (2011) 195-200 ISSN: 0210-1963 doi: 10.3989/arbor.2011.Extra-3n3144

\section{ENSEÑAR A GESTIONAR LA INNOVACIÓN EN EL MÁSTER}

\author{
F. J. Arcega \\ Grupo EduQTech, Universidad de Zaragoza \\ EINA, C/ María de Luna 3, Zaragoza \\ arcegafj@unizar.es \\ I. Plaza \\ C. T. Medrano \\ Grupo EduQTech, Universidad de Zaragoza \\ EUPT, Ciudad Escolar s/n, Teruel \\ iplaza@unizar.es \\ ctmedra@unizar.es
}

\section{TEACHING TO INNOVATE IN THE MASTER}

\begin{abstract}
One of the most important skills of a university master oriented to the research is innovation and one important aspect is how to manage it. For replaying to the needs demanded by the Society through the research and development programmes, technical personnel able to carry out such projects and manage it are needed. Universities have launched different programmes of teaching for covering such needs of technical knowledge but as well it is important to prepare the students to manage the research. In this paper, the experience of the authors of two years teaching the subject "Management of electronics research projects under quality criteria" in the Master of Electronics Engineering is presented with the aim of improving this skill in the students by the knowledge and practice of management processes of electronics projects of research, development and innovation.
\end{abstract}

KEY WORDS: Innovation; quality; R\&D\&I management.

\section{INTRODUCCIÓN}

La adaptación de las titulaciones al proceso de Bolonia conlleva la necesidad de impartir, además de contenidos, unas competencias para que los alumnos puedan desarrollar en su vida laboral los conocimientos adquiridos en la universidad y también que éstas puedan ser medidas al finalizar el proceso de aprendizaje. Según el Real Decreto 1393/2007, una de las competencias importantes en un máster es la capacidad de gestionar proyectos de investigación, tanto si el destino del titulado es la investigación como si es la empresa. Esta competencia es más importante aun cuando el máster está relacionado con la Tecnología
RESUMEN: Una de las competencias importantes de un máster universitario de introducción a la investigación es la innovación y un aspecto importante es cómo gestionarla. Para responder a las necesidades que la Sociedad demanda a través de los diversos programas de investigación y desarrollo que las distintas empresas lanzan, hace falta personal técnico capaz de llevar a cabo dichos proyectos y de gestionarlos bien. Las universidades han lanzado diversos programas de enseñanza para cubrir estas necesidades de conocimientos técnicos pero hace falta también dotar a los investigadores de formación adecuada en su gestión. En este artículo se presenta la experiencia de los autores tras dos años impartiendo la asignatura optativa "Gestión de proyectos de investigación electrónicos bajo criterios de calidad" en el Máster de Ingeniería Electrónica, con el objetivo de fomentar esta habilidad en los alumnos mediante el conocimiento y la práctica del proceso de gestión de los proyectos electrónicos de investigación, desarrollo e innovación tecnológica.

PALABRAS CLAVE: Innovación; calidad; gestión de I+D+i.

Electrónica, que es una de las ramas de mayor relevancia actual en la investigación, el desarrollo y la innovación tecnológica $(\mathrm{I}+\mathrm{D}+\mathrm{i})$.

En este artículo se presenta la experiencia de los autores tras los últimos dos cursos en los han impartido la asignatura optativa "Gestión de proyectos de investigación electrónicos bajo criterios de calidad" en el Máster Universitario de Ingeniería Electrónica de la Universidad de Zaragoza, así como su experiencia en actividades de investigación y gestión de sistemas de calidad y mejora continua (véase Plaza, 2009; Plaza, Arcega y otros, 2010 o en Plaza, Medrano y Posa, 2009). 
El presente trabajo se puede aplicar a otros másteres universitarios que deseen incluir en sus titulados las competencias relacionadas con la gestión de la $I+D+i$, no solo a los relacionados con la Electrónica.

Los egresados de un máster como el de Ingeniería Electrónica, disciplina del mayor interés científico-tecnológico, con muchos de departamentos dedicados a su enseñanza, con un elevado número de artículos científicos dedicados publicados en el área y con aplicación en la mayoría de los sectores industriales, deben tener la adecuada formación, tanto generalista como especializada, véase la memoria de verificación del máster de la Universidad de Zaragoza, 2011.

Otros ejemplos relacionados con este tema los podemos encontrar en las asignaturas de "Procesos para la gestión de las TIC" o "Diseño de sistemas y metodologías" en el máster de Sistemas Electrónicos de la Universidad Politécnica de Madrid (como en UPMa, 2011) de 5 créditos cada una que han sido sustituidas por "Metodologías, calidad y habilidades personales" en el nuevo máster universitario en Ingeniería de Sistemas Electrónicos (como en UPMb, 2011) o la asignatura "Gestión de la transferencia y la innovación" en el máster en Ingeniería Electrónica de la Universidad Rovira y Virgili de 4 créditos (como en URV, 2011).

\section{Objetivos del máster y de la asignatura}

El principal objetivo de este trabajo consiste en presentar el diseño y puesta en marcha de una asignatura de carácter transversal e innovador en su contexto en un máster de corte tecnológico, comentando la experiencia de los docentes: enfoque dado a la asignatura, contenidos y criterios seguidos, metodología empleada, problemas encontrados, aspectos valorados como positivos, cuestiones a mejorar, etc.

\subsection{Estructura del máster}

El máster universitario en Ingeniería Electrónica se ha estructurado en dos grupos importantes de competencias técnicas dependiendo de la elección de los estudiantes: el bloque temático de "Sistemas de Potencia" y el bloque temático "Ambientes Inteligentes".
El máster consta de 60 créditos mínimo donde 20 corresponden al trabajo fin de máster, 30 al bloque de optatividad y 10 a materias básicas obligatorias para todos los estudiantes. Se oferta a 30 alumnos por curso a los que se pretende dar una base común de Electrónica Analógica, Digital y de Potencia con dos bloques de intensificación en Electrónica Industrial o en Electrónica para las TIC, pero todo ello orientado a la investigación desarrollo e innovación en Ingeniería Electrónica.

El máster contiene cursos y seminarios para dotar al alumno con las capacidades y metodologías necesarias para la realización de proyectos de investigación y una futura tesis doctoral. La formación de profesionales cualificados en la I+D+i de sistemas electrónicos responde a una demanda de las empresas que desarrollan su actividad en estos sectores, algunas de las cuales participan activamente en el máster impartiendo seminarios profesionales, ofertando becas de iniciación a la investigación y acogiendo estudiantes para realizar estancias durante el último período de su formación, véase la memoria del máster en UZ, 2009.

\subsection{La asignatura}

La asignatura de "Gestión de proyectos de investigación electrónicos bajo criterios de calidad" es de 4 créditos y, debido a su carácter transversal, voluntaria para los dos bloques de optatividad del máster. Los alumnos reciben 18 horas de clases magistrales, 10 para resolver problemas de casos en el aula y 12 horas de trabajo en laboratorio y se complementa con otra asignatura de optativa de 2 créditos cuyo nombre es "Seminarios de I+D+i", orientada a presentar experiencias de técnicos que realizan actividades de $I+D+i$ en las empresas y les cuentan su experiencia o bien profesores de la universidad y responsables de proyectos de investigación.

Con la asignatura se pretende que los alumnos aprendan a gestionar los proyectos y actividades de $\mathrm{I}+\mathrm{D}+\mathrm{i}$, en base a los requisitos normativos de la norma UNE 166001, 2006. En la asignatura se desarrollan aspectos como las consideraciones de diseño a tener en cuenta, la evaluación de la calidad del producto, los ensayos típicos previos a la comercialización o la propia gestión de la innovación.

Para lograr estas competencias, se han organizado unos contenidos basados en los conceptos básicos de gestión de un sistema de calidad, las consideraciones del diseño 
electrónico, el desarrollo y la evaluación de los proyectos, la evaluación de la calidad del producto electrónico y la gestión de la innovación, sus herramientas y la protección y explotación de resultados.

El perfil de los alumnos inscritos en la asignatura indica que en su mayoría son Ingenieros Técnicos (60\%) repartidos casi por igual entre los dos bloques de optatividad, están trabajando a la vez que hacen el máster (75\%) y tienen un conocimiento bajo o muy bajo (85\%) en calidad y sobre todo quieren aprender a gestionar proyectos y quieren tener conocimientos de calidad.

\section{Desarrollo}

La metodología empleada en la asignatura se basa en darles los conocimientos básicos necesarios y que en gran medida se encuentran en la normativa UNE 166.001, 2006 y UNE 166.002, 2006. Luego estos conocimientos se aplican a casos prácticos para que el alumno vaya asimilando estos contenidos y como se usan en proyectos concretos de I+D+i.

Para que los alumnos comprendan y apliquen desde el principio los conceptos recogidos en la normativa de gestión de proyectos se ha optado por darles desde el principio del curso el tema del trabajo que deben desarrollar como proyecto final de la asignatura. De esta manera cada clase y cada práctica desarrolla alguno de los aspectos a considerar y a practicar. De este modo los alumnos llegan a final con suficiente práctica para poder realizar la parte final del trabajo con no demasiado esfuerzo habida cuenta que en el máster van saturados y se juntan muchas actividades y exámenes al final del período lectivo.

Este último año se ha centrado en el desarrollo de un dispositivo electrónico para la enseñanza de código Braille. Así cada alumno tiene que realizar un proyecto que por término medio está saliendo por unas 50 páginas en las que se contemplan los aspectos de la normativa y que para garantizar una buena evaluación se recogen en una rúbrica, cuyos aspectos fundamentales se recogen en la tabla I.

En cada uno de los conceptos se han seguido los criterios marcados en la normativa correspondiente de gestión de proyectos de $\mathrm{I}+\mathrm{D}+\mathrm{i}$.
TABLA I: CONCEPTOS Y PESO RECOGIDO EN LA RÚBRICA DE EVALUACIÓN DEL TRABAJO FINAL

\begin{tabular}{|l|l|}
\hline \multicolumn{1}{|c|}{ Aspecto } & Peso \\
\hline Presentación & $10 \%$ \\
\hline Objetivos & $10 \%$ \\
\hline Estado del arte & $20 \%$ \\
\hline Planificación & $20 \%$ \\
\hline Ensayos & $10 \%$ \\
\hline Presupuesto & $20 \%$ \\
\hline Bibliografia & $10 \%$ \\
\hline
\end{tabular}

\section{Resultados}

Como trabajo de la asignatura se decidió el primer año que cada alumno trabajase sobre un proyecto de su interés. La realidad fue que algunos alumnos tardaron mucho tiempo en decidirse y cuando lo hicieron ya había pasado mucho tiempo del curso. Por otra parte, no se tenía una homogeneidad a la hora de corregir el trabajo ni de que algunos de los temas a considerar estuviesen incluidos y trabajados por el alumno. (Algunos eligieron temas de software y sin electrónica como trabajo, lo que dificultó mucho la aplicación de conceptos y su posterior evaluación del aprendizaje.)

El segundo año se ha optado por fijar un único tema de proyecto de $1+D+i$ sobre el que aplicar las enseñanzas. Desde el punto de vista del profesorado ha permitido desarrollar y aplicar todos los contenidos para todos los alumnos por igual, planificando las clases con claridad y tener una mayor homogeneidad a la hora de corregir y de valorar el trabajo de los alumnos y su aprendizaje. A pesar de todo, en las encuestas realizadas a los alumnos hemos encontrado alguna queja porque se les obliga a realizar e estado del arte de algo que no les gusta en algún caso.

En todos los casos, la evaluación se ha llevado a cabo de la siguiente manera. La evaluación de la parte teórica se ha hecho de modo independiente por cada profesor con un examen teórico de tipo test, pero la parte del trabajo se ha evaluado por los tres profesores de modo independiente y las tres calificaciones obtenidas por cada alumno son bastante coincidentes. Bien es cierto, que para la corrección se ha hecho uso de las rúbricas, donde se reflejan 
los criterios a evaluar según los apartados indicados en la sección anterior.

También se han llevado a cabo encuestas de satisfacción, tanto globales por parte del máster, como individuales en la asignatura. En ambos casos los resultados obtenidos muestran una buena satisfacción de los alumnos, aunque la mejor satisfacción es que los alumnos matriculados en el máster eligen de modo mayoritario la asignatura. En el curso 2009/2010 hubo 14 alumnos matriculados en la asignatura y 17 en el 2010/2011.

Los alumnos que aprueban en junio, en la primera convocatoria y el primer año en que se imparte, son 10 de los 14 matriculados, un aprobado, 8 notables y 1 sobresaliente. Los resultados son razonables ya que la encuesta global marca que han ido muy saturados de trabajo con todas las materias y han ido dejando algunas asignaturas para la segunda convocatoria o para el año siguiente en el caso de alumnos que trabajan. En el segundo año y en la primera convocatoria, son 11 los alumnos que aprueban de los 17 matriculados, dos aprobados, 8 notables y 1 sobresaliente. El número de no presentados ha crecido de 4 a 6 .

Como aspecto negativo cabe señalar que el horario de la asignatura es bastante incómodo al tener todos los cursos horario los viernes de 15 a 17 horas para las clases de teoría y de 18 a 20 horas de los viernes para las prácticas. A pesar de todo la matrícula y la asistencia son bastante buenas.

La figura 1 muestra los resultados de la encuesta de satisfacción realizada en la asignatura a la que han respondido 14 alumnos. Se han analizado cinco aspectos considerados importantes y los grados de medida han sido:

D: Deficiente, S: Suficiente, B: Bien, MB: Muy bien, E: Excelente

Como puede apreciarse, no ha habido ninguna respuesta que considere deficiente ninguno de los aspectos analizados y la mayoría de los alumnos consideran que los cinco aspectos analizados han resultado de forma muy bien o excelente.

De los cinco aspectos considerados, lo mejor valorado resulta ser la puntualidad. También el material docente suministrado y la adecuación de los contenidos. Una de las razones pensamos que los primeros que aplicamos los criterios de calidad y mejora continua somos los tres profesores de la asignatura al estar inmersos en el grupo de investigación, véase (EduOTech, 2011).

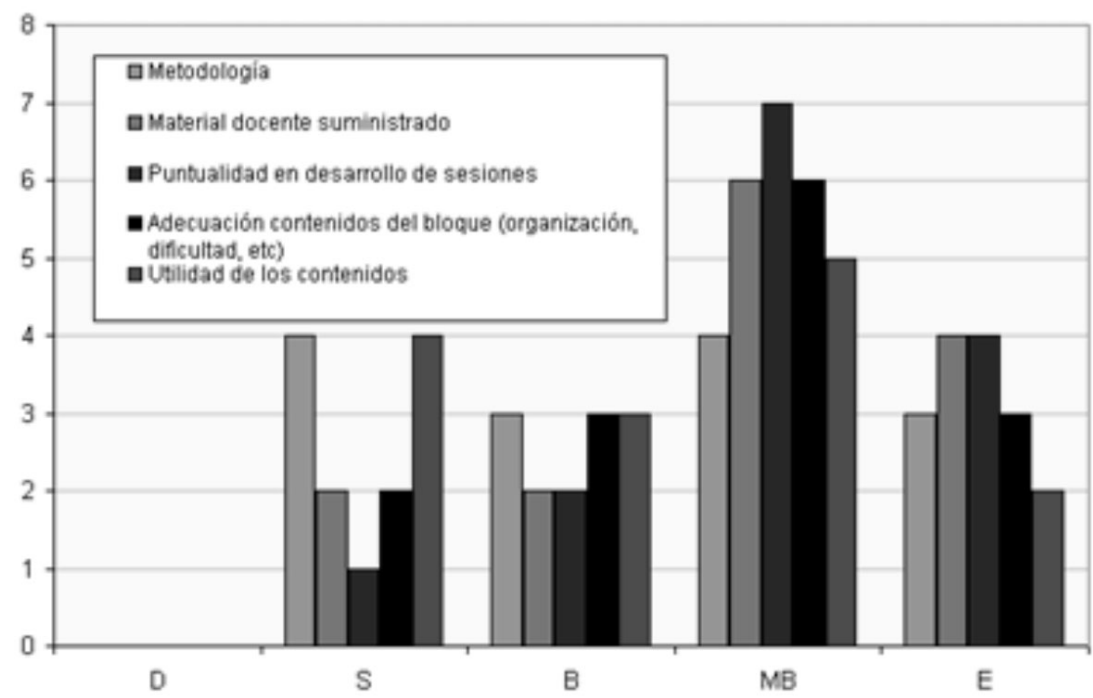

Figura 1. Resultados de la encuesta de la asignatura. 
En metodología y utilidad de contenidos ha habido cuatro alumnos que solo consideran suficiente lo realizado en la asignatura. Pensamos que ello se debe a su bajo conocimiento de los conceptos de calidad y mejora continua y también porque el primer año se perdió mucho tiempo hasta que algunos alumnos decidieron cuál era el tema de investigación sobre el que iban a desarrollar el trabajo final de la asignatura.

En la figura 2 se muestran los resultados de la encuesta general realizada a todos los alumnos del máster. Los resultados varian desde muy insatisfecho (MI) a muy satisfecho (MS) y se han dejado dos campos más para el caso de no aplica NA o que no han respondido a la encuesta (blanco). Los alumnos que contestan la encuesta general son 22,12 Ingenieros Técnicos Industriales, 4 Ingenieros Industriales, 3 Ingenieros de Telecomunicación y los 3 restantes otros titulados.

La encuesta la han respondido 22 alumnos y podemos considerar que más del $50 \%$ de los alumnos se muestran satisfechos o muy satisfechos con la titulación en general y que ha visto cubiertas sus expectativas iniciales. La percepción del alumno es que la formación recibida se considera de utilidad para su futuro profesional e incluso más del 50\% de los encuestados recomendaría este máster a futuros estudiantes.

El perfil de los alumnos es muy variado a pesar de los créditos de homogeneización porque se destinan a formación básica en Electrónica pero cada titulación imprime carácter y la orientación hacia la investigación es bastante diferente según la titulación de procedencia. Esto hace que algunos alumnos no tengan el interés deseado por el profesorado de la asignatura.

\section{Conclusiones}

Se ha incluido una asignatura transversal en el Máster de Ingeniería Electrónica para que los alumnos aprendan a gestionar la innovación en los proyectos de ingeniería Electrónica bajo criterios de calidad y mejora continua, y siguiendo la normativa española sobre el tema.

Se destaca como muy positivo disponer de un tema de proyecto sobre el que aplicar los conocimientos de la asignatura desde el primer día. Aunque haya habido quejas por el tema elegido el segundo año, presenta ventajas

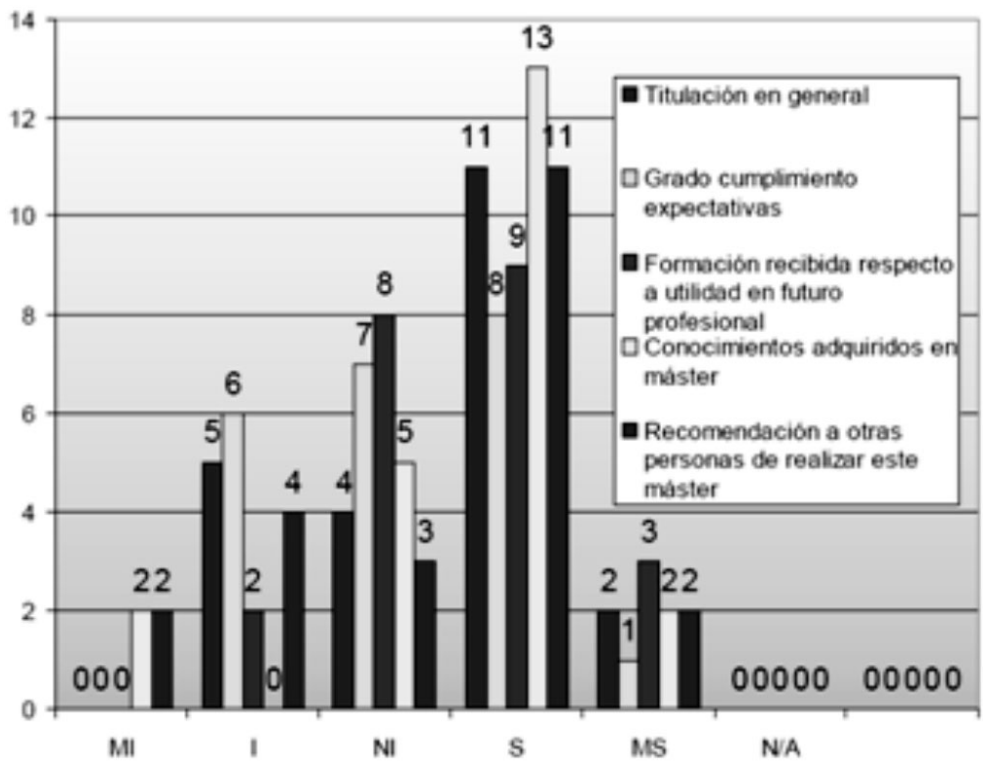

Figura 2. Resultados de la encuesta del máster. 
a los profesores por unificar los trabajos y los alumnos tienen la ventaja de trabajar desde el primer día sobre un tema concreto y aplicar los conceptos que se van desarroIlando en la asignatura. La solución de compromiso para lograr mayor interés en los alumnos sería el elegir dos temas uno de cada uno de los bloques de intensificación del máster y en colaboración con profesores de las otras asignaturas.

La asignatura debería ser obligatoria para todos los alumnos del máster, para que todos tengan la competencia de gestión de proyectos de $I+D+i$ porque estas actividades también necesitan, como todo, una buena base para que el proceso de innovación no sea fortuito sino que los efectos de la mejora continua hagan que la labor de innovar sea fructífera y continuada.

Se espera que las promociones que vayan saliendo sean más competitivas al gestionar mejor los proyectos de $\mathrm{I}+\mathrm{D}+\mathrm{i}$ de Electrónica que realicen en su futuro profesional. Ya han pasado dos promociones por la asignatura y los resultados y experiencia obtenidos han sido positivos por parte de los tres docentes y por parte de la mayoría de los estudiantes.

\section{BIBLIOGRAFÍA}

AENOR (2006): UNE 166.001, "Gestión de la I+D+i: Requisitos de un proyecto de I+D+i".

AENOR (2006): UNE 166002, "Gestión de $\mathrm{I}+\mathrm{D}+\mathrm{i}$. Requisitos para los sistemas de gestión de la I+D+i".

EduQTech (2011): http://www.unizar.es/ eduqtech/ (último acceso 20-07-2011).

Plaza, I. (2010): "Innovación ¿También en educación?" "Inovação! Também em educação?", Editorial IEEE-RITA, vol. 5, n. ${ }^{\circ} 4$, noviembre.

Plaza, I. y Arcega, F. et al. (2009): "Gestión de la I+D+i en grupos universitarios. Propuesta de actuación", Forum Calidad, n. ${ }^{\circ} 206$, pp. 47-53.

Plaza, I.; Medrano, C. y Posa, A. B. (2009): "Calidad en actividades de I+D+i. Aplicación en el sector TIC". RC Libros.

RD 1393/2007 por el que se establecen las enseñanzas universitarias oficiales. Ministerio de Educación y Ciencia.
UZ (2009): Memoria de verificación del título oficial de máster universitario en Ingeniería Electrónica.

UZ (2011): Máster universitario en Ingeniería Electrónica (último acceso 20-072011) http://www.unizar.es/mie/index. htm

UPMa (2011): Máster universitario en Sistemas Electrónicos (último acceso 2007-2011) http://mse.die.upm.es/index. php?status=programa

UPMb (2011): Máster universitario en Ingeniería de los Sistemas Electrónicos (último acceso 20-07-2011) http://mise.die.upm.es/index.php? status=programa

URV (2011): Máster universitario en Ingeniería Electrónica (último acceso 20-07-2011) http://www.urv.cat/masters_oficials/es_pla_master_eng_ electronica_it_recercall_60.html 